\title{
Morphologic patterns and treatment of transplant glomerulopathy: A retrospective analysis
}

\author{
Rui Abreu $^{1}$ (D) | Fernanda Carvalho ${ }^{2}$ | Helena Viana ${ }^{2}$ | Isabel Mesquita $^{2}$ | \\ Marília Possante $^{2}$ | Inês Aires ${ }^{2}$ | Fernando Caeiro ${ }^{2}$ | Cecília Silva ${ }^{2}$ | \\ Patrícia Cotovio $^{2}$ | Aníbal Ferreira ${ }^{2}$ | Francisco Remédio ${ }^{2}$ | Fernando Nolasco ${ }^{2}$
}

\author{
${ }^{1}$ Nephrology Department, Centro Hospitalar \\ Trás-os-Montes e Alto Douro, Vila Real, \\ Portugal \\ ${ }^{2}$ Nephrology Department, Hospital Curry \\ Cabral, Lisboa, Portugal

\section{Correspondence} \\ Rui Abreu, Nephrology Department, Centro \\ Hospitalar Trás-os-Montes e Alto Douro, Vila \\ Real, Portugal. \\ Email: ruimigabreu@gmail.com
}

\begin{abstract}
Transplant glomerulopathy is mainly due to chronic antibody-mediated rejection and actually represents a major cause of long-term allograft failure. The lack of effective treatment remains a serious problem in transplantation. A retrospective and uni-center study was performed in 48 kidney allograft recipients with transplant glomerulopathy between January 2010 and December 2015. Median time for diagnosis was 7.1 (3.611.8) years post-transplant. Light microscopy showed severity of transplant glomerulopathy in the majority of patients (cg1=10.4\%; $\operatorname{cg} 2=20.8 \%$; $\operatorname{cg} 3=68.8 \%$ ). Moderate microvascular inflammation was present in $56.3 \%$ (g+ptc $\geq 2$ ), and almost half of recipients (51.1\%) were $C 4 d$ positive in immunofluorescence. Female gender $(P=.001)$, age $(P=.043)$, renal dysfunction $(P=.002)$, acute rejection episodes $(P=.026)$, and anti-HLA class II antibodies $(P=.004)$ were associated with kidney allograft failure. Treatment of transplant glomerulopathy was performed in $67.6 \%$ of patients. The histologic and laboratory features that led to a therapeutic intervention were score ptc $(P=.021), C 4 d$ $(P=.03)$, and the presence of anti-HLA antibodies $(P=.029)$, whereas score ah $(P=.005)$ was associated with conservative measure. The overall cumulative kidney allograft survival at 10 years was $75 \%$. Treatment of transplant glomerulopathy was ineffective to improve long-term kidney allograft survival.
\end{abstract}

\section{KEYWORDS}

chronic allograft rejection, histopathology, immunosupression, transplant glomerulopathy, treatment

\section{1 | INTRODUCTION}

Transplant glomerulopathy is a morphologic pattern of chronic kidney allograft damage and actually represents a major cause of long-term allograft failure. ${ }^{1,2}$ This entity results from endothelial remodeling after sustained antibody-mediated injury, which leads to multilayering and double contours of basement membrane. ${ }^{1}$ Most common causes for transplant glomerulopathy are chronic antibody-mediated rejection, thrombotic microangiopathy, and $\mathrm{HCV}$ infection. ${ }^{1}$ The prevalence of transplant glomerulopathy secondary to chronic antibody-mediated rejection varies from $5 \%$ to $20 \%{ }^{2}$ According to revised classification of chronic active antibody-mediated rejection, three features should be present for diagnosis: morphologic evidence of chronic lesions, evidence of antibody interaction with microcirculation, and presence of donor specific antibodies (DSA) of HLA or other antigens. ${ }^{3}$ Clinical manifestations often include hypertension, kidney allograft dysfunction, and proteinuria. ${ }^{1}$ Unfortunately, there is a lack of effective treatment for transplant glomerulopathy due to its chronic and irreversible injuries. ${ }^{1}$ Thus, prevention and early detection of this entity are two crucial approaches. ${ }^{1}$ Usual therapeutic regimens are intravenous immunoglobulin, plasmapheresis, immunoadsorption, splenectomy, and more recently rituximab, bortezomib, and eculizumab. ${ }^{1,4}$ Similar strategies are used also for desensitization protocols. 
Despite clinical impact of this entity, very few studies have been concerned with respective treatment options. ${ }^{2}$ The aim of this study was to analyze clinico-pathological data and therapeutic management in kidney allograft recipients with transplant glomerulopathy.

\section{2 | MATERIALS AND METHODS}

A retrospective and uni-center study was performed in kidney allograft recipients with transplant glomerulopathy diagnosed between January 2010 and December 2015. We identified 48 cases. The diagnosis of transplant glomerulopathy and chronic allograft-mediated rejection was made by presence of structural evidence of chronic tissue injury (cg score $>0$ ) and evidence of recent antibody interaction with vascular endothelium (C4d positivity by immunofluorescence technique or moderate microvascular inflammation defined by score $\mathrm{g}+\mathrm{ptc} \geq 2$ ) or presence of DSA detected using solid phase assays. Continuous variables were expressed in median and interquartile range and were compared by Mann-Whitney $U$ test. Proportions were used for categorical data and compared by chi-square test. The major outcome was kidney allograft survival and was analyzed by KaplanMeier method.

\section{3 | RESULTS}

Median age of kidney allograft recipients was of 51 (41-57) years, and $62.5 \%$ was of male gender. Hypertension, dyslipidemia, and diabetes were present in $97.3 \%, 62.2 \%$, and $18.9 \%$, respectively. Patients received a deceased kidney transplant in $88.6 \%$. Nineteen percent were sensitized recipients, and $10.8 \%$ of patients had a previous kidney transplant. Induction immunosuppressive therapy was represented by thymoglobulin (65\%) and basiliximab (20\%). No monoclonal antibodies were used in $15 \%$. Calcineurin inhibitors were present in almost maintenance immunosuppressive regimens (70.6\%) in association with antimetabolite and corticosteroids. Median time from transplant to biopsy diagnosis was 7.1 (3.6-11.8) years. Median follow-up time after kidney transplant was 8.2 (5.8-13.7) years, and the mean follow-up time from biopsy to outcome was of $1.3 \pm 1.4$ years (minimum: 0.01; maximum: 5.2 years). Serum creatinine and proteinuria at the time of biopsy were $2.0(1.6-2.8) \mathrm{mg} / \mathrm{dL}$ and 1.6 (0.7-3.1) $\mathrm{g} / 24 \mathrm{~h}$, respectively. Anti-HLA class I or II antibodies were present in $48.4 \%$ and $35.5 \%$, with MFIs of 5114 (2722-9068). DSAs were detected in $48.4 \%$. Light microscopy showed severity of transplant glomerulopathy in the majority of patients (cg1=10.4\%; cg2=20.8\%; cg3=68.8\%). Interstitial fibrosis and tubular atrophy (IF/TA) were noted in $83.3 \%$ and $81.2 \%$, respectively. Moderate microvascular inflammation was present in $56.3 \%$ ( $\mathrm{g}+\mathrm{ptc} \geq 2$ ). Almost half of recipients (51.1\%) were C4d positive in immunofluorescence (Table 1). A negative correlation was observed between HLA compatibilities and score ptc $(r=-.4, P=.018)$. In univariate analysis, female gender $(P=.001)$, age $(P=.043)$, renal dysfunction $(P=.002)$, acute rejection episodes $(P=.026)$, and anti-HLA class II antibodies $(P=.004)$ were associated
TABLE 1 Baseline characteristics

\begin{tabular}{|lc|}
\hline Baseline characteristics & \\
\hline Male gender & $62.5 \%$ \\
\hline Age & $51(41-57)$ \\
\hline Hypertension & $97.3 \%$ \\
\hline Diabetes & $18.9 \%$ \\
\hline Dyslipidemia & $62.2 \%$ \\
\hline HCV infection & $16.7 \%$ \\
\hline Cause of renal disease & \\
\hline Glomerulonephritis & $31.8 \%$ \\
\hline Chronic NTI & $13.6 \%$ \\
\hline ADPKD & $6.8 \%$ \\
\hline Hypertensive nephrosclerosis & $6.8 \%$ \\
\hline Diabetic nephropathy & $4.5 \%$ \\
\hline Others & $9.1 \%$ \\
\hline Uncertain & $27.3 \%$ \\
\hline Donor's age & $47(44-58)$ \\
\hline Previous transplant & $10.8 \%$ \\
\hline Sensitization & $19.0 \%$ \\
\hline PRA & $18(0-26)$ \\
\hline Compatibilities & $2(1-3)$ \\
\hline Acute rejection & $60.7 \%$ \\
\hline Creatinine & $2.0(1.6-2.8)$ \\
\hline Proteinuria & $1.6(0.7-3.1)$ \\
\hline Anti-HLA class I & $48.4 \%$ \\
\hline Anti-HLA class II & $35.5 \%$ \\
\hline MFI & $5114(2722-9068)$ \\
\hline Microvascular inflammation & \\
\hline C4d & \\
\hline
\end{tabular}

with kidney allograft failure (Table 2). No histopathologic differences were seen between patients with renal loss and those with a functioning allograft. Kidney allograft biopsy was performed at similar time in two groups ( 7.1 vs 8.7 years, $P=.330$ ). Mean follow-up time from diagnosis was slightly superior in allograft survival group (1.7 vs $0.7, P=.044)$, but without differences between groups in respect to median follow-up time after kidney transplant (9.6 vs $7.7, P=.540$; Table 2).

Treatment of transplant glomerulopathy was performed in $67.6 \%$ of patients, based on multiple immunosuppressive regimens (Table 3). Rituximab ( $n=16)$ and intravenous immunoglobulin (IVIG) $(n=13)$ were the most common drugs administrated. Other regimens used were methylprednisolone $(n=9)$, increase of maintenance immunosuppression (IS) ( $n=9)$, tacrolimus ( $n=6)$, everolimus $(n=3)$, and plasmapheresis ( $n=3$; Figure 1 ). The histologic and laboratory features that led to a therapeutic intervention were score ptc $(P=.021)$, C4d $(P=.03)$, and the presence of anti-HLA antibodies $(P=.029)$, whereas score ah $(P=.005)$ was associated with a conservative measure. The overall cumulative kidney allograft survival at 10 years was 
TABLE 2 Predictive factors of kidney allograft failure

\begin{tabular}{|c|c|c|c|}
\hline Predictive factors & $\begin{array}{l}\text { Kidney allograft survival } \\
(n=24)\end{array}$ & $\begin{array}{l}\text { Kidney allograft failure } \\
(n=14)\end{array}$ & $P$-value \\
\hline Male gender & $83.3 \%$ & $42.9 \%$ & .01 \\
\hline Age & 50 & 56 & .043 \\
\hline Hypertension & $100 \%$ & $92.3 \%$ & .168 \\
\hline Diabetes & $12.5 \%$ & $30.8 \%$ & .176 \\
\hline Dyslipidemia & $79.2 \%$ & $30.8 \%$ & .004 \\
\hline HCV infection & $8.3 \%$ & $33.3 \%$ & .058 \\
\hline Donor's age & 46 & 54 & .181 \\
\hline Previous transplant & $16.7 \%$ & $0 \%$ & .119 \\
\hline Sensitization & $16.7 \%$ & $22.7 \%$ & .748 \\
\hline PRA & $16 \%$ & $22 \%$ & .702 \\
\hline Compatibilities & 2 & 2 & .663 \\
\hline Acute rejection & $50 \%$ & $100 \%$ & .026 \\
\hline Creatinine & 1.8 & 2.8 & .002 \\
\hline Proteinuria & 1.2 & 2.0 & .063 \\
\hline Anti-HLA class I & $63.6 \%$ & $11.1 \%$ & .004 \\
\hline Anti-HLA class II & $18.2 \%$ & $77.8 \%$ & \\
\hline MFI & 4863 & 7987 & .495 \\
\hline $\begin{array}{l}\text { Microvascular } \\
\text { inflammation }\end{array}$ & $58.3 \%$ & $71.4 \%$ & .501 \\
\hline C4d & $50 \%$ & $46.2 \%$ & 1.0 \\
\hline IF/TA & $75 \%$ & $85.7 \%$ & .435 \\
\hline Time to diagnosis & 7.1 & 8.7 & .330 \\
\hline $\begin{array}{l}\text { Follow-up time (from } \\
\text { biopsy) }\end{array}$ & 1.7 & 0.7 & .044 \\
\hline $\begin{array}{l}\text { Follow-up time (from } \\
\text { transplant) }\end{array}$ & 9.6 & 7.7 & .540 \\
\hline
\end{tabular}

TABLE 3 Immunosuppressive treatment combinations in transplant glomerulopathy

\begin{tabular}{|l|l|}
\hline Treatment combinations & $\mathbf{n}$ \\
\hline Rituximab + IVIG & 4 \\
\hline Increase of immunosuppression (IS) & 3 \\
\hline Plasmapheresis + Rituximab + IVIG & 2 \\
\hline Rituximab + IVIG + Tacrolimus & 2 \\
\hline Rituximab + Methylprednisolone & 2 \\
\hline Methylprednisolone + Increase of IS & 2 \\
\hline Everolimus & 2 \\
\hline Plasmapheresis + Rituximab + IVIG + Tacrolimus + Increase of IS & 1 \\
\hline Rituximab + IVIG + Methylprednisolone + Everolimus & 1 \\
\hline Rituximab + IVIG + Methylprednisolone + Increase of IS & 1 \\
\hline Rituximab + IVIG + Methylprednisolone + Tacrolimus & 1 \\
\hline Methylprednisolone + Tacrolimus + Increase of IS & 1 \\
\hline Rituximab + Tacrolimus + Increase of IS & 1 \\
\hline IVIG + Methylprednisolone & 1 \\
\hline Rituximab & 1 \\
\hline
\end{tabular}

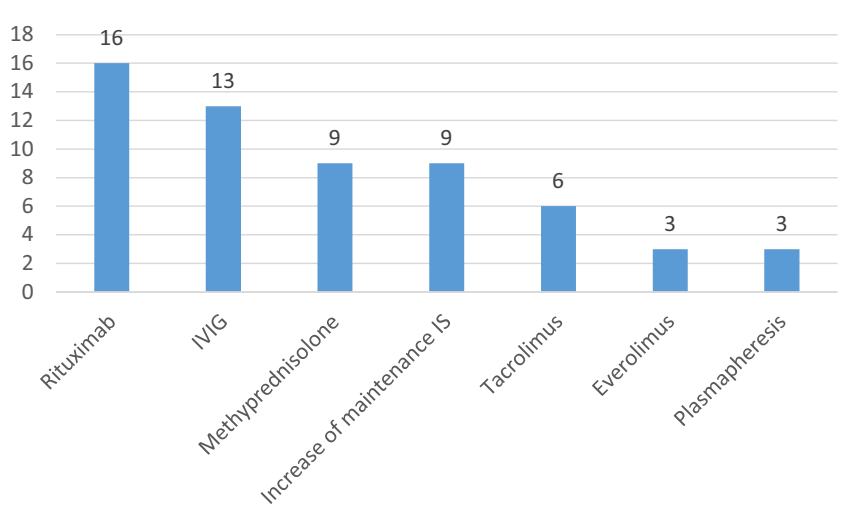

FIGURE 1 Immunosuppressive drugs

of $75 \%$ and $36.8 \%$ of kidney allograft failed at the end of follow-up. Treatment of transplant glomerulopathy proved ineffective to improve long-term kidney allograft survival (log rank=0.975; Figure 2). The administration of rituximab and IVIG alone or in combination did not improve either of the major outcomes (log rank=0.628; Figure 3). 


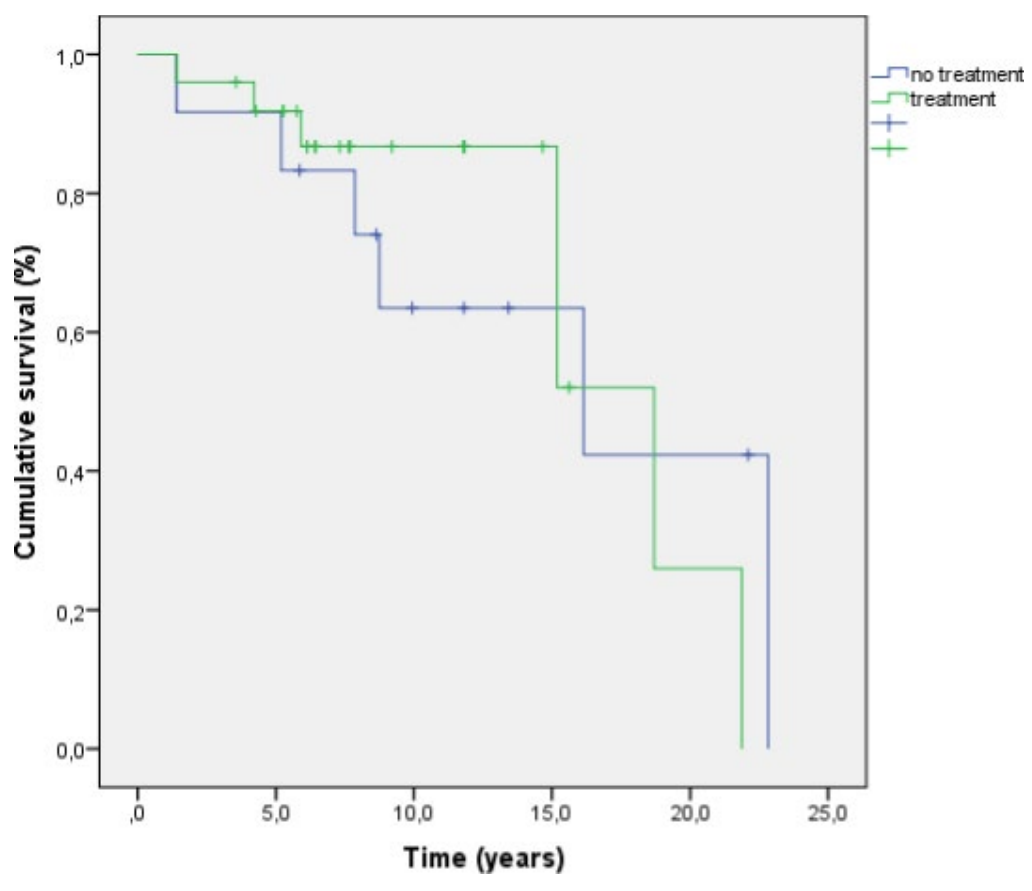

Subjects at risk:

\begin{tabular}{|l|c|c|c|c|c|c|}
\hline No treatment & 12 & 11 & 8 & 8 & 7 & 6 \\
\hline Treatment & 25 & 23 & 22 & 22 & 19 & 18 \\
\hline
\end{tabular}

FIGURE 2 Cumulative survival in transplant glomerulopathy according to treatment

\section{DISCUSSION}

In our study, median time for diagnosis was 7.1 (3.6-11.8) years and median follow-up time after kidney transplant was 8.2 (5.8-13.7) years. Thus, it seems likely that histologic confirmation of transplant glomerulopathy was performed too late, compared to other studies. This is due to most of kidney allograft biopsies being performed by clinical indication such as elevated creatinine and proteinuria. As seen in light microscopy, $89.6 \%$ had moderate to severe degree of transplant glomerulopathy and $56.3 \%$ had moderate microvascular inflammation. Different data were observed in Toki et al. ${ }^{5}$ study, with fewer chronicity in light microscopy (cg1=56\%; cg2=29\%; and cg3=15\%), probably due to earlier diagnosis (5.7 years after transplant). Similar results were seen in the Sis et al. ${ }^{6}$ report, in which kidney allograft biopsies (5.5 years after transplant) showed $35.8 \%$ mild, $45.3 \%$ moderate, and $18.9 \%$ severe disease.

Age and female gender were both risk factors for kidney allograft dysfunction and loss in our study. Some laboratory findings have also been recognized as predictors of kidney allograft failure. Elevated serum creatinine was associated with allograft loss as demonstrated in Redfield et al.'s ${ }^{7}$ study. Proteinuria also impacts in allograft survival, and our study demonstrated a trend toward higher proteinuria values in patients with failed allograft. ${ }^{8}$ Previous acute rejection episodes and anti-HLA class II antibodies were associated with worst outcome as mentioned in other studies. ${ }^{1,9}$ In contrast with few series, microvascular inflammation and C4d did not show significant relationship with kidney allograft failure. ${ }^{1,6,10}$

Treatment of transplant glomerulopathy remains a hard challenge in kidney transplantation. It should be initiated as early as possible before development of chronic and irreversible histopathologic changes. ${ }^{2}$ In our study, $67.6 \%$ received immunosuppression therapy. Rituximab $(n=16)$, intravenous immunoglobulin $(n=13)$, and methylprednisolone $(n=9)$ were the most common agents used. Histopathologic features such as score ptc, C4d, and the presence of anti-HLA antibodies induced to a therapeutic intervention, as seen in other studies. ${ }^{11}$ Dean et al. ${ }^{12}$ proved a correlation between early inflammation (molecular and ptc scores) and subsequent graft loss. On the other hand, score ah was associated with a conservative attitude. In our study, treatment of transplant glomerulopathy was ineffective to improve kidney allograft survival, probably due to severe degree of transplant glomerulopathy (median cg score $=3$ ) and high prevalence of IF (83.3\%) and TA (81.2\%). The same results were found in the Kamal et al. ${ }^{8}$ study, with no statistical differences between groups in respect to medical intervention. Cg score from 0 to 3 was 10\%, 48.1\%, 17.3\%, and 15.4\%, respectively, but $94 \%$ of patients had signs of IF/TA in kidney biopsies. Similar findings were seen in the Patri et al. ${ }^{13}$ study, with no difference in allograft failure between patients who received or not antirejection therapy. They also described a high prevalence of chronicity, with cg3 score of $42 \%$ and IF/TA of $89 \%$ and $90 \%$, respectively. Opposite to last reports, 


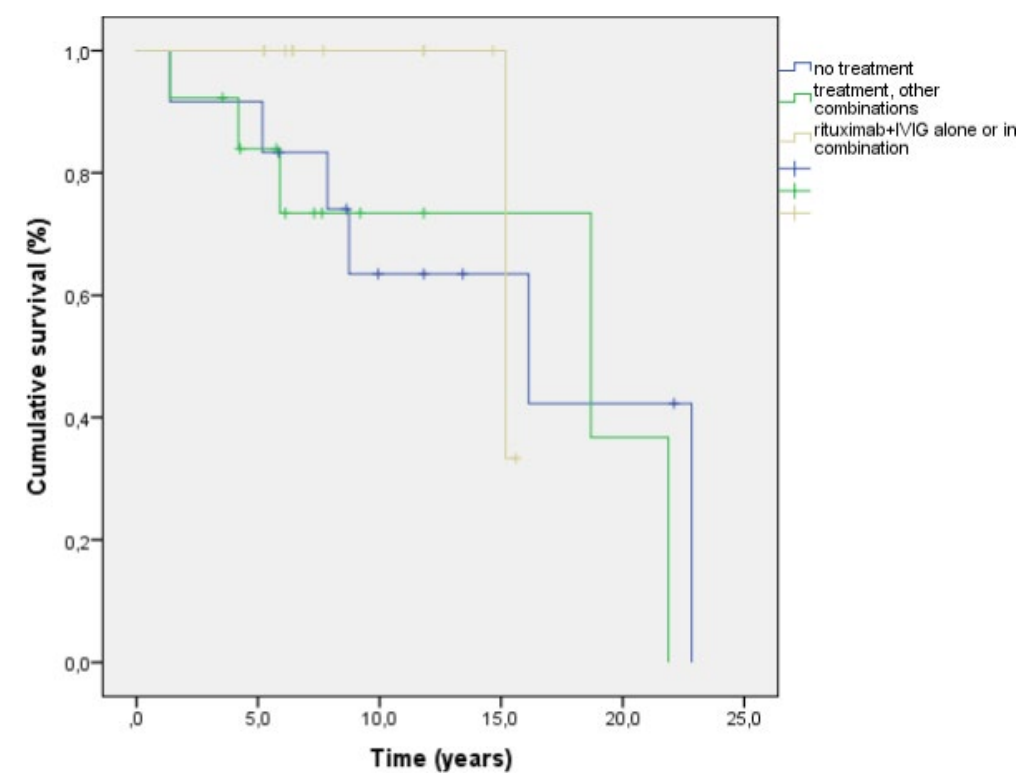

FIGURE 3 Cumulative survival in transplant glomerulopathy according to different treatment regimens

Subjects at risk:

\begin{tabular}{|l|c|c|c|c|c|c|}
\hline No treatment & 12 & 11 & 8 & 8 & 7 & 6 \\
\hline Other treatments & 13 & 11 & 10 & 10 & 9 & 8 \\
\hline Rituximab + IVIG & 12 & 12 & 12 & 12 & 10 & 10 \\
\hline
\end{tabular}

Kim et al. ${ }^{14}$ showed some effective response of combination therapy but only in nine patients with chronic antibody-mediated rejection. In Redfield et al.'s ${ }^{7}$ study, almost all recipients (93\%) were treated for chronic antibody-mediated rejection. Better survival rates were reached in treatment group, but kidney biopsies in that study showed less irreversible lesions (median cg score $=2) .{ }^{7}$ However, kidney allograft failure was superior compared to our study (76\% vs $36.8 \%$ ), but with higher follow-up time after transplantation (9.5 vs 8.2 years) and time after diagnosis (4.3 vs 1.3 ). ${ }^{7}$ Finally, in the Has and Mirocha report, patients with DSA and score ptc $+\mathrm{g} \geq 2$ on early biopsies treated for antibody-mediated rejection (plasmapheresis, IVIG, and/or rituximab) presented a trend toward lower incidence of transplant glomerulopathy. ${ }^{15}$ Thus, kidney biopsies should be performed as soon as possible because treatment of subclinical rejection in early stages seems to delay development of chronicity and progression to renal failure.

We recognize that our study is not without limitations. The first one is the retrospective study design, which restricts the relationship of causality between variables and the major outcome; second, the relatively small sample; and third, unfortunately few missing data of major outcome and respective therapy management $(n=10)$ from patients transferred to other hospital centers and from only one case without treatment information. Nonetheless, we thought that the topic of treatment inefficacy in advanced transplant glomerulopathy is a strong and unique message.

In conclusion, female gender, age, renal dysfunction, acute rejection episodes and anti-HLA class II antibodies constituted predictors for kidney allograft loss. The ptc score, anti-HLA antibodies, and presence of C4d in immunofluorescence seem to be important features to start immunosuppressive therapy. Treatment of transplant glomerulopathy did not improve long-term kidney allograft survival in this study.

\section{CONFLICT OF INTEREST}

None to declare.

\section{REFERENCES}

1. Husain S, Sis B. Advances in the understanding of transplant glomerulopathy. Am J Kidney Dis. 2013;62:352-363.

2. Remport A, Ivanyi B, Mathe Z, Tinckam K, Mucsi I, Molnar MZ. Better understanding of transplant glomerulopathy secondary to chronic antibody-mediated rejection. Nephrol Dial Transplant. 2015;30:1825-1833.

3. Haas M, Sis B, Racusen LC, et al. Banff 2013 meeting report: inclusion of c4d-negative antibody-mediated rejection and antibodyassociated arterial lesions. Am J Transplant. 2014;14:272-283.

4. Waiser J, Budde K, Schütz M, et al. Comparison between bortezomib and rituximab in the treatment of antibody-mediated renal allograft rejection. Nephrol Dial Transplant. 2012;27:1246-1251.

5. Toki $D$, Inui $M$, Ishida $H$, et al. Interstitial fibrosis is the critical determinant of impaired renal function in transplant glomerulopathy. Nephrology. 2016;21(Suppl 1):20-25.

6. Sis B, Campbell PM, Mueller T, et al. Transplant glomerulopathy, late antibody-mediated rejection and the $A B C D$ tetrad in kidney allograft biopsies for cause. Am J Transplant. 2007;7:1743-1752.

7. Redfield RR, Ellis TM, Zhong W, et al. Current outcomes of chronic active antibody mediated rejection - a large single center retrospective 
review using the updated BANFF 2013 criteria. Hum Immunol. 2016;77:346-352

8. Kamal L, Broin PO, Bao Y, et al. Clinical, histological, and molecular markers associated with allograft loss in transplant glomerulopathy patients. Transplantation. 2015;99:1912-1918.

9. Loupy A, Vernerey D, Tinel C, et al. Subclinical rejection phenotypes at 1 year post-transplant and outcome of kidney allografts. J Am Soc Nephrol. 2015;26:1721-1731.

10. Naesens M, Kuypers DRJ, De Vusser K, et al. The histology of kidney transplant failure: a long-term follow-up study. Transplantation. 2014;98:427-435.

11. Kahwaji J, Najjar R, Kancherla D, et al. Histopathologic features of transplant glomerulopathy associated with response to therapy with intravenous immune globulin and rituximab. Clin Transplant. 2014;28:546-553.

12. Dean PG, Park WD, Cornell LD, Schinstock CA, Stegall MD. Early subclinical inflammation correlates with outcomes in positive crossmatch kidney allografts. Clin Transplant. 2016;30:925-933.
13. Patri $\mathrm{P}$, Seshan SV, Matignon $\mathrm{M}$, et al. Development and validation of a prognostic index for allograft outcome in kidney recipients with transplant glomerulopathy. Kidney Int. 2016;89:450-458.

14. Kim MG, Kim YJ, Kwon HY, et al. Outcomes of combination therapy for chronic antibody-mediated rejection in renal transplantation. Nephrology. 2013;18:820-826.

15. Haas M, Mirocha J. Early ultrastructural changes in renal allografts: correlation with antibody-mediated rejection and transplant glomerulopathy. Am J Transplant. 2011;11:2123-2131.

How to cite this article: Abreu R, Carvalho F, Viana H, et al. Morphologic patterns and treatment of transplant glomerulopathy: A retrospective analysis. Clin Transplant. 2017;31:e12915. https://doi.org/10.1111/ctr.12915 